\title{
An Analytic Bolometric Light Curve Model of Interaction-Powered Supernovae and its Application to Type IIn Supernovae
}

\author{
Takashi J. Moriya ${ }^{1,2,3 \star}$, Keiichi Maeda ${ }^{1}$, Francesco Taddia ${ }^{4}$, Jesper Sollerman $^{4}$, \\ Sergei I. Blinnikov ${ }^{5,6,7}$, and Elena I. Sorokina ${ }^{7}$ \\ ${ }^{1}$ Kavli Institute for the Physics and Mathematics of the Universe (WPI), Todai Institutes for Advanced Study, University of Tokyo, \\ 5-1-5 Kashiwanoha, Kashiwa, Chiba 277-8583, Japan \\ 2 Department of Astronomy, Graduate School of Science, University of Tokyo, 7-3-1 Hongo, Bunkyo, Tokyo 113-0033, Japan \\ ${ }^{3}$ Research Center for the Early Universe, Graduate School of Science, University of Tokyo, \\ 7-3-1 Hongo, Bunkyo, Tokyo 113-0033, Japan \\ 4 The Oskar Klein Centre, Department of Astronomy, Stockholm University, AlbaNova, 10691 Stockholm, Sweden \\ ${ }^{5}$ Institute for Theoretical and Experimental Physics, Bolshaya Cheremushkinskaya 25, 117218 Moscow, Russia \\ ${ }^{6}$ Novosibirsk State University, Novosibirsk 630090, Russia \\ 7 Sternberg Astronomical Institute, M.V.Lomonosov Moscow State University, Universitetski pr. 13, 119992 Moscow, Russia
}

Accepted 2013 July 24. Received 2013 July 24; in original form 2013 March 24

\begin{abstract}
We present an analytic model for bolometric light curves which are powered by the interaction between supernova ejecta and a dense circumstellar medium. This model is aimed at modeling Type IIn supernovae to determine the properties of their supernova ejecta and circumstellar medium. Our model is not restricted to the case of steady mass loss and can be applied broadly. We only consider the case in which the optical depth of the unshocked circumstellar medium is not high enough to affect the light curves. We derive the luminosity evolution based on an analytic solution for the evolution of a dense shell created by the interaction. We compare our model bolometric light curves to observed bolometric light curves of three Type IIn supernovae (2005ip, 2006jd, 2010jl) and show that our model can constrain their supernova ejecta and circumstellar medium properties. Our analytic model is supported by numerical light curves from the same initial conditions.
\end{abstract}

(C) 2013 RAS 
Key words: circumstellar matter — stars: mass-loss — supernovae: general — supernovae: individual: SN 2005ip — supernovae: individual: SN 2006jd — supernovae: individual: SN 2010j1

\section{INTRODUCTION}

Massive stars which die as supernovae (SNe) do not end their lives as they were born. They change their mass, size, temperature, luminosity and many other properties during their evolution toward their death. The final fate, or the SN type, of a massive star is determined by these changes during their evolution (e.g., Heger et al. 2003).

One of the most critical factors which dramatically change the properties of a star and largely affect its final fate is mass loss. Massive stars continue to lose mass until the end of their lives because of their huge luminosities. From X-ray and radio observations of young $\mathrm{SNe}$, it has been possible to estimate the mass-loss rates of SN progenitors shortly before their explosions (e.g., Chevalier, Fransson, \& Nymark 2006; Chevalier \& Fransson 2006; Maeda 2013). In most cases, the estimated mass-loss rates are within the range expected from the radiation-driven mass loss (e.g., Owocki, Gayley, \& Shaviv 2004).

However, there are a number of SNe which seem to be strongly affected by the interaction with circumstellar media (CSM) whose densities are too high to be explained by the standard radiation-driven mass loss. Most of them are spectroscopically classified as Type IIn because of the narrow hydrogen emission lines seen in their spectra (Schlegel 1990; Filippenko 1997). The mass-loss rates of the progenitors before their explosions are estimated to be $\sim 10^{-4}-\sim 0.1 M_{\odot} \mathrm{yr}^{-1}$ (e.g., Taddia et al. 2013; Kiewe et al. 2012; Fox et al. 2011). Because of the high-density CSM, a cool dense shell is suggested to be created between SN ejecta and CSM (e.g., Chevalier \& Fransson 1994; Chugai et al. 2004). The cool dense shell can create dust grains efficiently and SNe IIn are promising sites for dust formation (e.g., Kozasa et al.|2009). SNe IIn can also be used as a distance ladder thanks to the dense shell (e.g., Blinnikov et al. 2012; Potashov et al. 2013). In addition, some SNe IIn can be observed at very high redshifts and may provide us with information about star formation and initial-mass functions in the early Universe (e.g., Cooke 2008; Cooke et al. 2009, 2012; Tanaka et al. 2012; Tanaka, Moriva, \& Yoshida 2013; Whalen et al. 2013).

Despite the many interesting phenomena associated with SNe IIn, the nature of SNe IIn

^ takashi.moriya@ipmu.jp 
is still not well-understood. Little is known about their progenitors or the mechanisms that cause such extreme mass loss just before the explosions. There are several possible SN progenitors suggested to cause extreme mass loss before the explosions, e.g., super-asymptoticgiant-branch (AGB) stars (e.g., Poelarends et al.|2008; Botticella et al.|2009) and massive red supergiants (RSGs) (e.g., Smith. Hinkle, \& Ryde 2009; Yoon \& Cantiello 2010). The progenitor of Type IIn SN 2008S which was found in archival images is actually consistent with a super-AGB star (Prieto et al. 2008). The small degree of association between SN IIn sites and $\mathrm{H} \alpha$ emitting regions within their host galaxies also seem consistent with these relatively low-mass progenitors (Anderson et al. 2012, but see also Crowther 2013). However, the other SN IIn progenitors detected in archival images are very massive stars which are rather consistent with luminous blue variables (LBVs) (e.g., Gal-Yam \& Leonard 2009; Mauerhan et al. 2013; Pastorello et al. 2013, see also Smith et al. 2011a.,b). LBVs are theoretically interpreted as an evolutionary stage to a Wolf-Rayet star and they have not been considered as a pre-SN phase (e.g., Crowther 2007; Maeder \& Mevnet 2000, but see Groh, Meynet, \& Ekström 2013; Moriya, Groh, \& Meynet 2013; Langer 2012). In addition, extreme mass-loss mechanisms of such very massive stars are not well-known. There are several suggested mechanisms to induce extreme mass loss, like pulsational pair-instability (e.g., Wooslev, Blinnikov, \& Heger 2007), rotation (e.g., Maeder \& Desjacaues 2001), porosity (e.g., Owocki, Gayley, \& Shaviv 2004), gravity-mode oscillations (e.g., Quataert \& Shiode 2012), or binary interaction (e.g., Chevalier 2012), but we still do not know which mechanisms are actually related to SNe IIn (see also Dwarkadas 2011).

For a better understanding of SNe IIn, especially their progenitors and extensive massloss mechanisms, we need more theoretical investigation of SNe IIn as well as more observational data. In this paper we develop a simple analytic bolometric LC model which can be used to fit SN IIn observations to estimate CSM and SN ejecta properties. We believe that the information obtained by applying our LC model to many SNe IIn can lead to a better understanding of SNe IIn.

This paper is organized as follows. We present our analytic LC model in Section 2, We derive the evolution of the shocked shell analytically and use it to obtain the bolometric LC evolution. We apply it to some observational SN IIn bolometric LCs and obtain constraints on the properties of SN ejecta and CSM for these SNe in Section 3, The discussion is given in Section 4 and we conclude this paper in Section 5 . 


\section{ANALYTIC BOLOMETRIC LIGHT CURVE MODEL}

In this section, we develop an analytic SN bolometric LC model under the assumption that its main power source is the kinetic energy of SN ejecta colliding with a dense CSM. At first, we analytically investigate the evolution of the dense shell created by the interaction in Section 2.1. The analytic solution for the evolution of the dense shell before time $t_{t}$ (see below) is essentially the same as obtained in previous works (e.g., Chevalier 1982a, 1990; Chevalier \& Fransson 1994, 2003) but our solution does not assume steady mass loss (see also Fransson, Lundqvist, \& Chevalier 1996).

After deriving the evolution of the dense shell, we provide an analytic expression for bolometric LCs. This method was introduced by Chugai \& Danziger (1994) (see also Wood-Vasey, Wang, \& Ald 2004; Svirski, Nakar, \& Sari 2012) to explain the luminoisity due to the interaction but their model assumes a CSM from steady mass loss. We generalize this method for the cases of nonsteady mass loss and apply our model to entire bolometric LCs. Chatzopoulos, Wheeler, \& Vinko (2012); Chatzopoulos et al. (2013) also follow a similar approach to obtain an analytic LC model from the interaction but they consider the case where the unshocked CSM is optically thick. Here, we consider the case in which the unshocked CSM does not affect the bolometric LC so much. Some SNe IIn are suggested to have very optically thick CSM to explain their huge luminosities (e.g., Chevalier \& Irwin 2011; Moriya \& Tominaga 2012; Moriva et al. 2013b; Ginzburg \& Balberg 2012) but they are beyond the scope of this paper. High energy photons are expected to be emitted when the CSM is optically thin (e.g., Chevalier \& Fransson 1994) but they are presumed to be absorbed by the dense shell because of its high column density and re-emitted as optical photons which are mainly observed (e.g., Wilms, Allen, \& McCray 2000). The inverse Compton scattering and other effects can also reduce the energy of the photons (e.g., Chevalier \& Irwin 2012).

\subsection{Evolution of the Shocked Dense Shell}

\subsubsection{General Case}

The shocked dense CSM and SN ejecta form a thin dense shell because of the efficient radiative cooling. We assume that the thickness of the shocked shell is much smaller than its radius and it can be denoted by a radius $r_{\mathrm{sh}}(t)$. The conservation of momentum requires $M_{\mathrm{sh}} \frac{d v_{\mathrm{sh}}}{d t}=4 \pi r_{\mathrm{sh}}^{2}\left[\rho_{\mathrm{ej}}\left(v_{\mathrm{ej}}-v_{\mathrm{sh}}\right)^{2}-\rho_{\mathrm{csm}}\left(v_{\mathrm{sh}}-v_{\mathrm{w}}\right)^{2}\right]$ 
where $M_{\mathrm{sh}}$ is the total mass of the shocked SN ejecta and CSM, $v_{\mathrm{sh}}$ is the velocity of the shell, $\rho_{\mathrm{ej}}$ is the SN ejecta density, $v_{\mathrm{ej}}$ is the SN ejecta velocity, $\rho_{\mathrm{csm}}$ is the CSM density, and $v_{\mathrm{w}}$ is the CSM velocity. We derive the evolution of $r_{\mathrm{sh}}$ based on this equation. We do not use the equation for the conservation of energy which is necessary to derive the self-similar solution including the reverse shock and the forward shock (Chevalier 1982b; Nadyozhin 1985). This is because of the radiative energy loss from the dense shell. When the radiative cooling is efficient, the shocked region does not extend as wide as the width expected from the selfsimilar solution due to the loss of the thermal pressure caused by the radiative energy loss. Thus, our approximation to neglect the shell width is presumed to be a good approximation to the shell evolution.

We further assume that the CSM density follows $\rho_{\mathrm{csm}}=D r^{-s}$ and that the CSM velocity $v_{\mathrm{w}}$ is constant. We adopt a double power-law profile for the density of homologously $\left(v_{\mathrm{ej}}=\right.$ $r / t$ ) expanding SN ejecta $\left(\rho_{\mathrm{ej}} \propto r^{-n}\right.$ outside and $\rho_{\mathrm{ej}} \propto r^{-\delta}$ inside) based on numerical simulations of SN explosions (e.g., Matzner \& McKee 1999). With SN kinetic energy $E_{\text {ej }}$ and SN ejecta mass $M_{\mathrm{ej}}$, the SN density structure is expressed as

$\rho_{\mathrm{ej}}\left(v_{\mathrm{ej}}, t\right)= \begin{cases}\frac{1}{4 \pi(n-\delta)} \frac{\left[2(5-\delta)(n-5) E_{\mathrm{ej}}\right]^{(n-3) / 2}}{\left[(3-\delta)(n-3) M_{\mathrm{ej}}\right]^{(n-5) / 2}} t^{-3} v_{\mathrm{ej}}^{-n} & \left(v_{\mathrm{ej}}>v_{t}\right), \\ \frac{1}{4 \pi(n-\delta)} \frac{\left[2(5-\delta)(n-5) E_{\mathrm{ej}}\right]^{(\delta-3) / 2}}{\left[(3-\delta)(n-3) M_{\mathrm{ej}}\right]^{(\delta-5) / 2}} t^{-3} v_{\mathrm{ej}}^{-\delta} & \left(v_{\mathrm{ej}}<v_{t}\right),\end{cases}$

where $v_{t}$ is obtained from the density continuity condition at the interface of the two density structures as well as $E_{\mathrm{ej}}$ and $M_{\mathrm{ej}}$ as follows,

$v_{t}=\left[\frac{2(5-\delta)(n-5) E_{\mathrm{ej}}}{(3-\delta)(n-3) M_{\mathrm{ej}}}\right]^{\frac{1}{2}}$.

The outer density slope $n$ depends on the SN progenitor and $n \simeq 7$ ( $n=6.67$ exactly) is the lowest possible $n$ expected from the self-similar solution of Sakurai (1960) (e.g., Chevalier 1990). A value of $n \simeq 10$ is expected for SN Ib/Ic and SN Ia progenitors (Matzner \& McKee 1999; Kasen 2010) and $n \simeq 12$ is expected for explosions of RSGs (Matzner \& McKee 1999). The inner density slope $\delta$ is $\delta \simeq 0-1$.

At first, the outer SN ejecta with $\rho_{\mathrm{ej}} \propto r^{-n}$ starts to interact with the CSM. In this phase, $M_{\mathrm{sh}}$ becomes

$$
\begin{aligned}
M_{\mathrm{sh}} & =\int_{R_{\mathrm{p}}}^{r_{\mathrm{sh}}} 4 \pi r^{2} \rho_{\mathrm{csm}} d r+\int_{v_{\mathrm{ej}} / t}^{v_{\mathrm{ej}, \max } / t} 4 \pi r^{2} \rho_{\mathrm{ej}} d r, \\
& =\frac{4 \pi D}{3-s} r_{\mathrm{sh}}^{3-s}+\frac{t^{n-3}}{(n-\delta)(n-3) r_{\mathrm{sh}}^{n-3}} \frac{\left[2(5-\delta)(n-5) E_{\mathrm{ej}}\right]^{(n-3) / 2}}{\left[(3-\delta)(n-3) M_{\mathrm{ej}}\right]^{(n-5) / 2}},
\end{aligned}
$$


where $R_{\mathrm{p}}$ is the radius of the progenitor, $v_{\mathrm{ej}, \max }$ is the velocity of the outermost layer of the SN ejecta before the interaction. In deriving Equation (5) from Equation (41), we have assumed $r_{\mathrm{sh}} \gg R_{\mathrm{p}}, v_{\mathrm{ej}, \max } \gg v_{\mathrm{ej}}$, and $s<3$.

With the above equations and $v_{\mathrm{ej}}=r_{\mathrm{sh}} / t$ (homologous expansion of the SN ejecta), the equation for the conservation of momentum becomes

$$
\begin{aligned}
{\left[\frac{4 \pi D}{3-s} r_{\mathrm{sh}}^{3-s}+\right.} & \left.\frac{t^{n-3}}{(n-\delta)(n-3) r_{\mathrm{sh}}^{n-3}} \frac{\left[2(5-\delta)(n-5) E_{\mathrm{ej}}\right]^{(n-3) / 2}}{\left[(3-\delta)(n-3) M_{\mathrm{ej}}\right]^{(n-5) / 2}}\right] \frac{d^{2} r_{\mathrm{sh}}}{d t^{2}}= \\
& \frac{1}{(n-\delta)} \frac{\left[2(5-\delta)(n-5) E_{\mathrm{ej}}\right]^{(n-3) / 2} t^{n-3}}{\left[(3-\delta)(n-3) M_{\mathrm{ej}}\right]^{(n-5) / 2} r_{\mathrm{sh}}^{n-2}}\left(\frac{r_{\mathrm{sh}}}{t}-\frac{d r_{\mathrm{sh}}}{d t}\right)^{2}-4 \pi D r_{\mathrm{sh}}^{2-s}\left(\frac{d r_{\mathrm{sh}}}{d t}\right)^{2} .
\end{aligned}
$$

Here, we assume that the CSM velocity is much smaller than the shell velocity $\left(v_{\mathrm{sh}} \gg v_{\mathrm{w}}\right)$. Solving the differential equation, we get a power-law solution

$r_{\mathrm{sh}}(t)=\left[\frac{(3-s)(4-s)}{4 \pi D(n-4)(n-3)(n-\delta)} \frac{\left[2(5-\delta)(n-5) E_{\mathrm{ej}}\right]^{(n-3) / 2}}{\left[(3-\delta)(n-3) M_{\mathrm{ej}}\right]^{(n-5) / 2}}\right]^{\frac{1}{n-s}} t^{\frac{n-3}{n-s}}$.

Note that $r_{\mathrm{sh}}$ obtained with this approach has the same time dependence as the self-similar solution $\left[t^{(n-3) /(n-s)}\right]$ (Chevalier 1982a, b; Chevalier \& Fransson 2003; Nadyozhin 1985).

Equation (7) holds until the time $t_{t}$ when the interacting region reaches down to the inner ejecta, namely when the $v_{\text {ej }}$ entering the shell becomes $v_{t}$ or $r_{\mathrm{sh}}\left(t_{t}\right)=v_{t} t_{t}$ is satisfied, i.e.,

$t_{t}=\left[\frac{(3-s)(4-s)}{4 \pi D(n-4)(n-3)(n-\delta)} \frac{\left[(3-\delta)(n-3) M_{\mathrm{ej}}\right]^{(5-s) / 2}}{\left[2(5-\delta)(n-5) E_{\mathrm{ej}}\right]^{(3-s) / 2}}\right]^{\frac{1}{3-s}}$.

After $t_{t}$, the density structure of the SN ejecta entering the shell starts to follow $\rho_{\mathrm{ej}} \propto r^{-\delta}$ and the equation of the momentum conservation becomes

$$
\begin{aligned}
& {\left[\frac{4 \pi D}{3-s} r_{\mathrm{sh}}^{3-s}+\right.}\left.M_{\mathrm{ej}}-\frac{r_{\mathrm{sh}}^{3-\delta}}{(n-\delta)(3-\delta) t^{3-\delta}} \frac{\left[2(5-\delta)(n-5) E_{\mathrm{ej}}\right]^{(\delta-3) / 2}}{\left[(3-\delta)(n-3) M_{\mathrm{ej}}\right]^{(\delta-5) / 2}}\right] \frac{d^{2} r_{\mathrm{sh}}}{d t^{2}}= \\
& \frac{1}{(n-\delta)} \frac{\left[2(5-\delta)(n-5) E_{\mathrm{ej}}\right]^{(\delta-3) / 2} r_{\mathrm{sh}}^{2-\delta}}{\left[(3-\delta)(n-3) M_{\mathrm{ej}}\right]^{(\delta-5) / 2} t^{3-\delta}}\left(\frac{r_{\mathrm{sh}}}{t}-\frac{d r_{\mathrm{sh}}}{d t}\right)^{2}-4 \pi D r_{\mathrm{sh}}^{2-s}\left(\frac{d r_{\mathrm{sh}}}{d t}\right)^{2} .
\end{aligned}
$$

Generally, we cannot solve Equation (9) analytically but the solution of Equation (9) is expected to asymptotically approach the solution of the differential equations

$$
\begin{aligned}
M_{\mathrm{sh}} \frac{d^{2} r_{\mathrm{sh}}}{d t^{2}} & =4 \pi r_{\mathrm{sh}}^{2}\left(-\rho_{\mathrm{csm}} v_{\mathrm{sh}}^{2}\right) \\
\left(\frac{4 \pi D}{3-s} r_{\mathrm{sh}}^{3-s}+M_{\mathrm{ej}}\right) \frac{d^{2} r_{\mathrm{sh}}}{d t^{2}} & =-4 \pi D r_{\mathrm{sh}}^{2-s}\left(\frac{d r_{\mathrm{sh}}}{d t}\right)^{2} .
\end{aligned}
$$

The asymptotic solution from Equation (11) satisfies the equation

$$
\frac{4 \pi D}{4-s} r_{\mathrm{sh}}(t)^{4-s}+(3-s) M_{\mathrm{ej}} r_{\mathrm{sh}}(t)-(3-s) M_{\mathrm{ej}}\left(\frac{2 E_{\mathrm{ej}}}{M_{\mathrm{ej}}}\right)^{\frac{1}{2}} t=0 .
$$


The boundary conditions

$$
\begin{aligned}
r_{\mathrm{sh}}(t=0) & =0, \\
\frac{d r_{\mathrm{sh}}}{d t}(t=0) & =\left(\frac{2 E_{\mathrm{ej}}}{M_{\mathrm{ej}}}\right)^{\frac{1}{2}},
\end{aligned}
$$

are applied in deriving Equation (12). As the asymptotic solution is derived by assuming that most of the SN ejecta is in the dense shell, the dependence of $r_{\mathrm{sh}}(t)$ on the SN ejecta structure $(n$ and $\delta)$ disappears.

\subsubsection{Case of Steady Mass Loss ( $(=2)$}

Here, we write down $r_{\mathrm{sh}}(t)$ derived in the previous section for the special case of the steady mass loss $(s=2)$. The CSM density structure becomes

$\dot{M}=4 \pi r^{2} \rho_{\text {csm }} v_{\mathrm{w}}$,

where $\dot{M}$ is the mass-loss rate and $D$ can be expressed as

$D=\frac{\dot{M}}{4 \pi v_{\mathrm{w}}}$.

Then, $r_{\mathrm{sh}}(t)$ before $t=t_{t}$ is

$r_{\mathrm{sh}}(t)=\left[\frac{2}{(n-4)(n-3)(n-\delta)} \frac{\left[2(5-\delta)(n-5) E_{\mathrm{ej}}\right)^{(n-3) / 2}}{\left[(3-\delta)(n-3) M_{\mathrm{ej}}\right]^{(n-5) / 2}} \frac{v_{\mathrm{w}}}{\dot{M}}\right]^{\frac{1}{n-2}} t^{\frac{n-3}{n-2}} \quad\left(t<t_{t}\right)$,

and

$t_{t}=\frac{2}{(n-4)(n-3)(n-\delta)} \frac{\left[(3-\delta)(n-3) M_{\mathrm{ej}}\right]^{3 / 2}}{\left[2(5-\delta)(n-5) E_{\mathrm{ej}}\right]^{1 / 2}} \frac{v_{\mathrm{w}}}{\dot{M}}$.

The asymptotic solution after $t_{t}$ becomes

$r_{\mathrm{sh}}(t)=\frac{v_{\mathrm{w}}}{\dot{M}} M_{\mathrm{ej}}\left[-1+\left(1+2 \sqrt{\frac{2 E_{\mathrm{ej}}}{M_{\mathrm{ej}}^{3}}} \frac{\dot{M}}{v_{\mathrm{w}}} t\right)^{\frac{1}{2}}\right]$.

As noted in Section 2.1.1, the asymptotic solution is independent of the SN density structure $(n$ and $\delta$ ).

\subsection{Bolometric Light Curve}

\subsubsection{General Case}

We construct an analytic bolometric LC based on $r_{\mathrm{sh}}(t)$ obtained in the previous section. We assume that the kinetic energy of the SN ejecta is the dominant source of the SN luminosity. The available kinetic energy is 
$d E_{\mathrm{kin}}=4 \pi r_{\mathrm{sh}}^{2} \frac{1}{2} \rho_{\mathrm{csm}} v_{\mathrm{sh}}^{2} d r_{\mathrm{sh}}$,

and thus the bolometric luminosity will be

$L=\epsilon \frac{d E_{\mathrm{kin}}}{d t}=2 \pi \epsilon \rho_{\mathrm{csm}} r_{\mathrm{sh}}^{2} v_{\mathrm{sh}}^{3}$

where $\epsilon$ is the conversion efficiency from kinetic energy to radiation. Especially, the bolometric luminosity before $t_{t}$ can be expressed as a power-law function

$L=L_{1} t^{\alpha}$

where

$$
\begin{aligned}
& L_{1}=\frac{\epsilon}{2}(4 \pi D)^{\frac{n-5}{n-s}}\left(\frac{n-3}{n-s}\right)^{3}\left[\frac{(3-s)(4-s)}{(n-4)(n-3)(n-\delta)} \frac{\left[2(5-\delta)(n-5) E_{\mathrm{ej}}\right]^{(n-3) / 2}}{\left[(3-\delta)(n-3) M_{\mathrm{ej}}\right]^{(n-5) / 2}}\right]^{\frac{5-s}{n-s}}, \\
& \alpha=\frac{6 s-15+2 n-n s}{n-s} .
\end{aligned}
$$

In Figure 1, $\alpha$ is plotted as a function of $s$ for $n=12,10,7$.

After $t_{t}$, the asymptotic bolometric LC can be obtained based on the asymptotic radius evolution from Equation (12).

$$
\begin{aligned}
L & =2 \pi \epsilon \rho_{\mathrm{csm}} r_{\mathrm{sh}}^{2} v_{\mathrm{sh}}^{3} \\
& =2 \pi \epsilon D r_{\mathrm{sh}}^{2-s}\left[\frac{(3-s) M_{\mathrm{ej}}\left(\frac{2 E_{\mathrm{ej}}}{M_{\mathrm{ej}}}\right)^{\frac{1}{2}}}{4 \pi D r_{\mathrm{sh}}^{3-s}+(3-s) M_{\mathrm{ej}}}\right]^{3}
\end{aligned}
$$

\subsubsection{Case of Steady Mass Loss (s=2)}

In the case of steady mass loss $(s=2)$, we can use $4 \pi D=\dot{M} / v_{\mathrm{w}}$ and express $L$ before $t_{t}$ as

$$
\begin{aligned}
L & =\frac{\epsilon}{2} \frac{\dot{M}}{v_{\mathrm{w}}} v_{\mathrm{sh}}^{3} \\
& =\frac{\epsilon}{2}\left(\frac{\dot{M}}{v_{\mathrm{w}}}\right)^{\frac{n-5}{n-2}}\left(\frac{n-3}{n-2}\right)^{3}\left[\frac{2}{(n-4)(n-3)(n-\delta)} \frac{\left[2(5-\delta)(n-5) E_{\mathrm{ej}}\right]^{(n-3) / 2}}{\left[(3-\delta)(n-3) M_{\mathrm{ej}}\right]^{(n-5) / 2}}\right]^{\frac{3}{n-2}} t^{-\frac{3}{n-2}} .
\end{aligned}
$$

This equation is basically the same as obtained in previous studies (e.g., Chugai \& Danziger 1994; Wood-Vasey, Wang, \& Aldering 2004).

We can also express the asymptotic bolometric LC after $t_{t}$ using Equation (19).

$L=\frac{\epsilon}{2} \frac{\dot{M}}{v_{\mathrm{w}}}\left(\frac{2 E_{\mathrm{ej}}}{M_{\mathrm{ej}}}\right)^{\frac{3}{2}}\left[1+2 \frac{\dot{M}}{v_{w}}\left(\frac{2 E_{\mathrm{ej}}}{M_{\mathrm{ej}}^{3}}\right)^{\frac{1}{2}} t\right]^{-\frac{3}{2}}$.

By defining two parameters $a$ and $b$ as

$a=\frac{\epsilon}{2} \frac{\dot{M}}{v_{\mathrm{w}}}\left(\frac{2 E_{\mathrm{ej}}}{M_{\mathrm{ej}}}\right)^{\frac{3}{2}}$, 


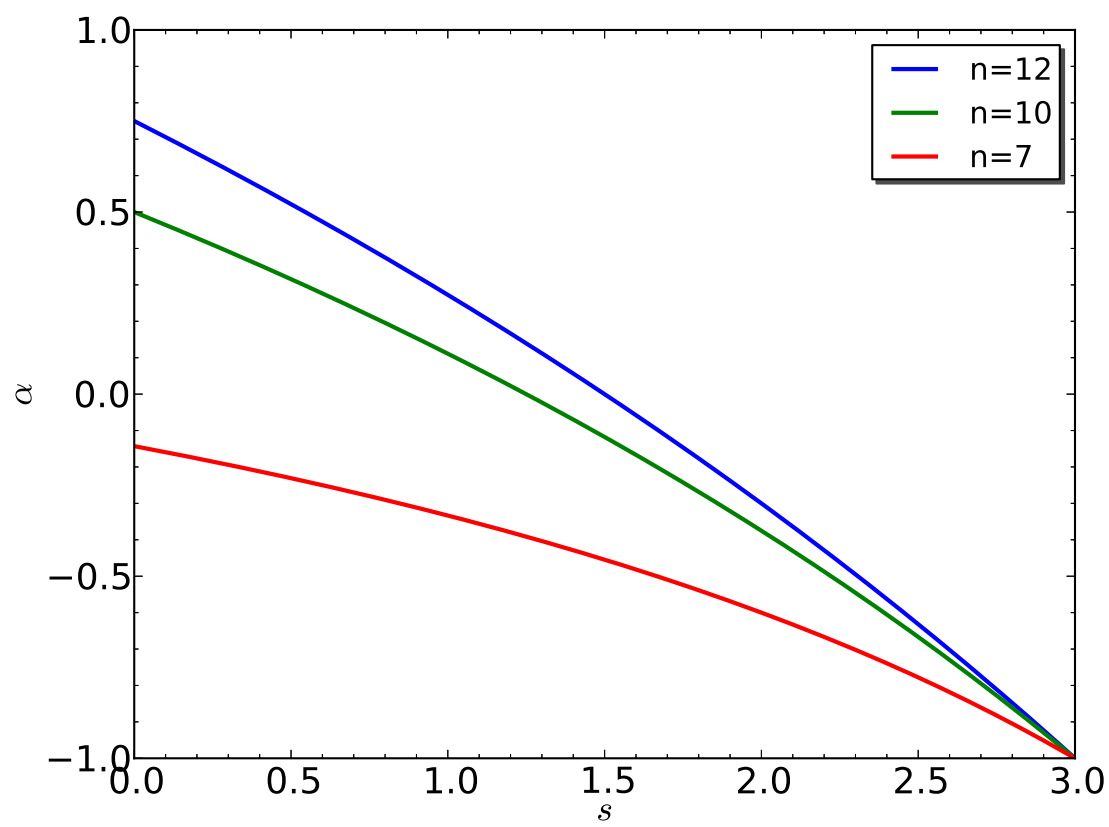

Figure 1. $\alpha\left(L \propto t^{\alpha}\right.$ before $\left.t_{t}\right)$ as a function of $s$ for some $n . n \simeq 12$ represents RSG explosions and $n \simeq 10$ is for $\mathrm{SNe} \mathrm{Ib} / \mathrm{Ic}$ and SNe Ia progenitors. $n \simeq 7$ is the minimum possible $n$.

$b=2 \frac{\dot{M}}{v_{\mathrm{w}}}\left(\frac{2 E_{\mathrm{ej}}}{M_{\mathrm{ej}}^{3}}\right)^{\frac{1}{2}}$,

we can express $L$ in a simple way. Namely,

$L=2^{-\frac{3(n-7)}{2(n-2)}} a b^{-\frac{3}{n-2}}\left(\frac{n-3}{n-2}\right)^{3} \frac{[2(5-\delta)(n-5)]^{\frac{3(n-3)}{2(n-2)}}}{[(n-4)(n-3)(n-\delta)]^{\frac{3}{n-2}}[(3-\delta)(n-3)]^{\frac{3(n-5)}{2(n-2)}}} t^{-\frac{3}{n-2}}$,

before $t_{t}$ and

$L=a(1+b t)^{-\frac{3}{2}}$,

long after $t_{t}$. Here, $t_{t}$ is expressed as

$t_{t}=\frac{4[(3-\delta)(n-3)]^{\frac{3}{2}}}{(n-4)(n-3)(n-\delta)[(5-\delta)(n-5)]^{\frac{1}{2}} b}$.

The physical parameters of CSM and SN ejecta have the relations

$$
\begin{aligned}
E_{\mathrm{ej}} & =\frac{2 a}{\epsilon b} \\
\frac{\dot{M}}{v_{\mathrm{w}}} M_{\mathrm{ej}}^{-\frac{3}{2}} & =\frac{1}{4}\left(\frac{\epsilon b^{3}}{a}\right)^{\frac{1}{2}} .
\end{aligned}
$$




\section{COMPARISON WITH OBSERVATIONS}

\subsection{Procedures}

We first show examples of procedures to fit our analytic bolometric LC to bolometric LCs constructed from observations. The actual processes for the comparison depend on the available information from observations but the basic concepts will be essentially the same as the examples presented here.

Our bolometric LC model consists of two components. Before $t_{t}$, the model LC has a power-law dependence on time $\left(L=L_{1} t^{\alpha}\right)$. Thus, we can first use the function $L_{1} t^{\alpha}$ to fit an early LC and obtain $L_{1}$ and $\alpha$. Assuming $n$, the CSM density slope $s$ can be constrained just by $\alpha$ through Equation (24) (Figure 1).

If there are spectral observations at these epochs and the shell velocity evolution can be estimated by them, we can use

$$
\begin{aligned}
v_{\mathrm{sh}}(t) & =\frac{d r_{\mathrm{sh}}}{d t} \\
& =\frac{n-3}{n-s}\left[\frac{(3-s)(4-s)}{4 \pi D(n-4)(n-3)(n-\delta)} \frac{\left[2(5-\delta)(n-5) E_{\mathrm{ej}}\right]^{(n-3) / 2}}{\left[(3-\delta)(n-3) M_{\mathrm{ej}}\right]^{(n-5) / 2}}\right]^{\frac{1}{n-s}} t^{-\frac{3-s}{n-s}} \\
& \equiv v_{1} t^{-\frac{3-s}{n-s}}
\end{aligned}
$$

to fit the velocity evolution and obtain $v_{1}$. Just from the three values, $L_{1}, \alpha$, and $v_{1}$, we can obtain the CSM density structure for given $\epsilon$ and $n$,

$D=\frac{1}{2 \pi \epsilon}\left(\frac{n-3}{n-s}\right)^{2-s} L_{1} v_{1}^{s-5}$

This means that we can estimate the mass-loss rate without assuming $M_{\mathrm{ej}}$ and $E_{\mathrm{ej}}$. As the time dependence of $v_{\text {sh }}$ is small, the velocity information of just a single epoch can constrain $D$. So far, $M_{\mathrm{ej}}$ and $E_{\mathrm{ej}}$ are degenerated and we have to assume either $M_{\mathrm{ej}}$ or $E_{\mathrm{ej}}$ to constrain the other parameter.

The formulae $L=L_{1} t^{\alpha}$ and $v_{\mathrm{sh}}=v_{1} t^{-(3-s) /(n-s)}$ can only be applied before $t_{t}$. After obtaining the physical values, we have to check whether $t_{t}$ is larger than the epochs used for the fitting. If there is an available bolometric LC after $t_{t}$, we can fit Equation (26) to the LC and obtain further constraints to break the degeneracy between $E_{\mathrm{ej}}$ and $M_{\mathrm{ej}}$.

We show how this procedure works in the next section by using actual bolometric LCs from observations.

(C) 2013 RAS, MNRAS 000, 1 29 
Table 1. SN IIn properties estimated by the bolometric LC model $(\epsilon=0.1)$.

\begin{tabular}{ccccc}
\hline SN & \multicolumn{2}{c}{$s\left(\rho_{\mathrm{csm}} \propto r^{-s}\right)$} & $\langle\dot{M}\rangle^{a}$ & $E_{\mathrm{ej}}$ \\
& $n=10$ & $n=12$ & $\left(M_{\odot} \mathrm{yr}^{-1}\right)$ & $\left(10^{51} \mathrm{erg}\right)$ \\
\hline 2005ip & 2.3 & 2.4 & $1.2-1.4 \times 10^{-3}$ & $13-15^{b}$ \\
$2006 \mathrm{jd}$ & 1.4 & 1.6 & $1.3-1.7 \times 10^{-3}$ & $12-13^{b}$ \\
$2010 \mathrm{j} 1$ & \multicolumn{2}{c}{$2.2^{b}$} & $0.039^{b}$ & $23^{b}$ \\
\hline
\end{tabular}

\footnotetext{
${ }^{a}$ Average rate derived by the CSM mass within $10^{16} \mathrm{~cm}$ and the CSM wind velocity $100 \mathrm{~km} \mathrm{~s}^{-1}$.

${ }^{b}$ Derived assuming $M_{\mathrm{ej}}=10 M_{\odot}$.
}

\subsection{Examples}

Here we compare our analytic bolometric LCs to observed LCs of SNe IIn 2005ip, 2006jd, and 2010jl, and estimate CSM and SN ejecta properties of them. We assume $\epsilon=0.1$ in this section. $\epsilon$ is affected by SN ejecta mass and CSM mass but it is typically of the order of 0.1 (e.g., Moriva et al. 2013b). All the fitting procedures in this section are performed by using the least-squares method unless otherwise mentioned. Table 1 is a summary of the SN IIn properties derived in this section.

\subsubsection{SN 2005ip}

SN 2005ip was intensively observed by Stritzinger et al. (2012) from ultraviolet to nearinfrared wavelengths. They derived a bolometric LC that we use for the comparison to our bolometric LC model. Optical photometric and spectroscopic observations are also reported by Smith et al. (2009), whereas Fox et al. (2009, 2010, 2011, 2013) summarize the nearinfrared observations of SN 2005ip. We assume that the explosion date of SN 2005ip was 9 days before its discovery and all the following dates are since the explosion.

At first, we fit the obtained bolometric LC up to 220 days by $L=L_{1} t^{\alpha}$ and we get $L=1.44 \times 10^{43}\left(\frac{t}{1 \text { day }}\right)^{-0.536} \operatorname{erg~s}^{-1}$.

In Figure 2 we show the result. $\alpha=-0.536$ corresponds to $s=2.3$ and $s=2.4$ for $n=10$ and $n=12$, respectively. Thus, the CSM around the progenitor of SN 2005ip likely had slightly steeper density structure than the expected density structure from steady mass loss. The deviation from the steady mass loss of SN IIn progenitors is also suggested from X-ray observations (Dwarkadas \& Gruszko 2012).

One interesting feature in Figure 2 is the similarity of the analytic LC from the SN ejecta-CSM interaction to the available energy from the radioactive decay of $0.18 M_{\odot}{ }^{56} \mathrm{Ni}$ 

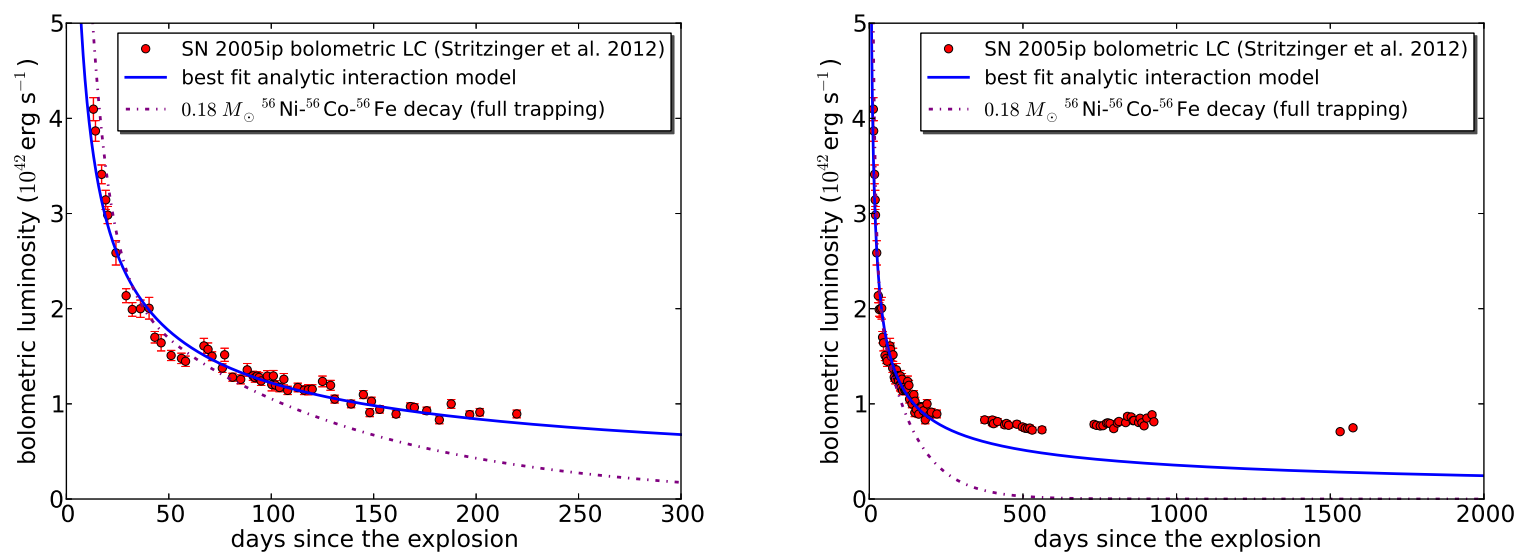

Figure 2. Bolometric LC of SN 2005ip (Stritzinger et al. 2012) and some LC models. The solid line is the best fit to $L=L_{1} t^{\alpha}$ up to 220 days. The dot-dashed line is the available energy from the radioactive decay of $0.18 M_{\odot}{ }^{56} \mathrm{Ni}$. The luminosity inputs from the two power souces resemble each other up to about 100 days and the later LC is required to distinguish between them.

before around 100 days. The available energy from the radioactive decay ${ }^{56} \mathrm{Ni} \rightarrow{ }^{56} \mathrm{Co} \rightarrow{ }^{56} \mathrm{Fe}$ is (Nadyozhin 1994)

$$
\left[6.45 \times 10^{43} e^{-t /(8.8 \text { days })}+1.45 \times 10^{43} e^{-t /(111.3 \text { days })}\right] \frac{M_{56} \mathrm{Ni}}{M_{\odot}} \operatorname{erg~s}^{-1},
$$

where $M_{56 \mathrm{Ni}}$ is the initial ${ }^{56} \mathrm{Ni}$ mass. We cannot distinguish between the two power sources only from the bolometric LC before about 100 days. The two energy sources can only be distinguished by LCs at later epochs. The similarity, especially at around 50 days, is because of the decay time of ${ }^{56} \mathrm{Co}$. At around 50 days, the radioactive energy from ${ }^{56} \mathrm{Co}$ is dominant and the available energy from the decay follows $\propto e^{-t /(111.3 \text { days })}$. The values and the decline rates (the first derivatives) of the functions following $\propto e^{-t /(111.3 \text { days })}$ and $\propto t^{-m}$ ( $m$ is a constant) can get similar at $t=111.3 m$ days. Looking at Figure $1, m \simeq 0.5$ at around $s \simeq 2$, so the two functions can be similar at around $t \simeq 50$ days. For a LC from the interaction to have a similar decline rate to that from the ${ }^{56}$ Co radioactive decay after $\simeq 100$ days, $m$ should be close to unity and the CSM density slope should be steep $(s \simeq 3$ ).

The shell velocity of SN 2005ip around 100 days since the explosion is likely $\simeq 17,500 \mathrm{~km} \mathrm{~s}^{-1}$ (Stritzinger et al. 2012). Then, based on Equation (40), we get

$\rho_{\mathrm{csm}}(r)=\left\{\begin{array}{lll}8.4 \times 10^{-16}\left(\frac{r}{10^{15} \mathrm{~cm}}\right)^{-2.3} \mathrm{~g} \mathrm{~cm}^{-3} & (n=10), \\ 1.0 \times 10^{-15}\left(\frac{r}{10^{15} \mathrm{~cm}}\right)^{-2.4} \mathrm{~g} \mathrm{~cm}^{-3} & (n=12) .\end{array}\right.$

The Thomson scattering optical depth $\tau_{\text {sh }}$ of the solar-metallicity unshocked CSM when the shell is at the radius $10^{15} \mathrm{~cm}$, above which the shell is located at the epochs we fit the LC, is 
$\tau_{\mathrm{sh}}= \begin{cases}0.22 & (n=10), \\ 0.25 & (n=12),\end{cases}$

and our assumption that the unshocked CSM does not affect the LC at the epochs we use for the fitting is justified. We estimate an average mass-loss rate $\langle\dot{M}\rangle$ by using the CSM mass within $10^{16} \mathrm{~cm}$. Assuming $v_{\mathrm{w}}=100 \mathrm{~km} \mathrm{~s}^{-1}$, the CSM mass within $10^{16} \mathrm{~cm}$ is lost from the progenitor in 32 years before the explosion. The average mass-loss rate in this period is $\langle\dot{M}\rangle=\left\{\begin{array}{lll}1.2 \times 10^{-3} & M_{\odot} \mathrm{yr}^{-1} & (n=10), \\ 1.4 \times 10^{-3} M_{\odot} \mathrm{yr}^{-1} & (n=12) .\end{array}\right.$

The bolometric luminosity of SN 2005ip after 300 days is almost constant $(\simeq 8.2 \times$ $10^{41} \mathrm{erg} \mathrm{s}^{-1}$ ). The asymptotic solution (Equation 26) can have a constant luminosity at a certain condition. For example, the asymptotic solution for $s=2$ (Equation 33) can be constant if $b t \ll 1$. However, for the case of SN 2005ip, we could not find a constant asymptotic solution which is consistent with the early LC before 300 days. The constant luminosity may be due to, e.g., another CSM component or light echos.

To constrain the SN properties, we assume $M_{\mathrm{ej}}=10 M_{\odot}$. Then, from $L_{1}$ above, we obtain

$E_{\mathrm{ej}}= \begin{cases}1.3 \times 10^{52} \mathrm{erg} \mathrm{s}^{-1} & (n=10), \\ 1.5 \times 10^{52} \mathrm{erg} \mathrm{s}^{-1} & (n=12) .\end{cases}$

$t_{t}$ becomes

$t_{t}= \begin{cases}4.2 \times 10^{3} \text { days } & (n=10), \\ 5.0 \times 10^{3} \text { days } & (n=12),\end{cases}$

so the epochs we used for the fitting ( $t<220$ days) are justified.

The average mass-loss rate we obtained $\left(10^{-3} M_{\odot} \mathrm{yr}^{-1}\right)$ is consistent with the rate estimated by Fox et al. $(2011)\left(1.8 \times 10^{-3} M_{\odot} \mathrm{yr}^{-1}\right)$ but larger than the rate suggested by Smith et al. (2009) $\left(2 \times 10^{-4} M_{\odot} \mathrm{yr}^{-1}\right)$. Based on these mass-loss rates, Smith et al. (2009) conclude that the progenitor of SN 2005ip is a massive RSG like VY CMa (e.g., Smith. Hinkle, \& Ryde 2009), while Fox et al. (2011) prefer a more massive progenitor like a LBV. Our results seem to support the latter scenario but depend on the value of $\epsilon$ assumed in deriving $D$ so we cannot constrain the progenitor strongly (see Section 4.1). In principle, we may be able to distinguish between the two progenitors with $n$, but our results are found not to depend much on $n$. Binary evolution may also be related to the dense CSM (e.g., Chevalier 2012). 


\subsubsection{SN 2006jd}

SN 2006jd was also observed in a wide spectral range by Stritzinger et al. (2012) and they obtained a bolometric LC. We use their bolometric LC for our modeling. We assume that the date of the explosion is 9 days before its discovery and the following dates are since the explosion. Chandra et al. (2012a) estimate CSM properties of SN 2006jd based on the X-ray and radio observations after about 400 days since explosion. They conclude that the CSM density profile is rather flat $(s \simeq 1.5-1.6)$ and the CSM density is $\sim 10^{6} \mathrm{~cm}^{-3}$ at $\sim 2 \times 10^{16} \mathrm{~cm}$. Fox et al. (2011, 2013) estimate the mass-loss rate based on near-infrared observations $\left(2.8 \times 10^{-3} M_{\odot} \mathrm{yr}^{-1}\right)$.

By fitting the LC before 250 days with $L=L_{1} t^{\alpha}$, we obtain

$L=3.87 \times 10^{42}\left(\frac{t}{1 \text { day }}\right)^{-0.0708} \operatorname{erg~s}^{-1}$.

From $\alpha=-0.0708$ obtained by the bolometric LC fitting, we obtain $s=1.4$ and $s=1.6$ for $n=10$ and $n=12$, respectively. The shell velocity of SN 2006jd around 100 days since the explosion is likely $\simeq 15,000 \mathrm{~km} \mathrm{~s}^{-1}$ (Stritzinger et al. 2012). Then, based on Equation (40), we get

$\rho_{\mathrm{csm}}(r)=\left\{\begin{array}{lll}2.6 \times 10^{-16}\left(\frac{r}{10^{15} \mathrm{~cm}}\right)^{-1.4} \mathrm{~g} \mathrm{~cm}^{-3} & (n=10), \\ 4.8 \times 10^{-16}\left(\frac{r}{10^{15} \mathrm{~cm}}\right)^{-1.6} \mathrm{~g} \mathrm{~cm}^{-3} & (n=12) .\end{array}\right.$

The Thomson scattering optical depth $\tau_{\text {sh }}$ of the solar-metallicity unshocked CSM when the shell is at the radius $10^{15} \mathrm{~cm}$ is

$\tau_{\mathrm{sh}}= \begin{cases}0.22 & (n=10), \\ 0.26 & (n=12),\end{cases}$

and our model is self-consistent. We estimate an average mass-loss rate by using the CSM mass within $10^{16} \mathrm{~cm}$ and $v_{\mathrm{w}}=100 \mathrm{~km} \mathrm{~s}^{-1}$ as we did for SN 2005ip in the previous section. The average mass-loss rate is

$\langle\dot{M}\rangle=\left\{\begin{array}{lll}1.3 \times 10^{-3} M_{\odot} \mathrm{yr}^{-1} & (n=10), \\ 1.7 \times 10^{-3} M_{\odot} \mathrm{yr}^{-1} & (n=12) .\end{array}\right.$

The estimated average mass-loss rate is consistent with the rate derived by Fox et al. (2011) from dust emission $\left(2.8 \times 10^{-3} M_{\odot} \mathrm{yr}^{-1}\right)$. Interestingly, the mass-loss rate is very close to those of SN 2005ip estimated in the previous section, although the density slopes are quite different $(s=2.3-2.4$ for SN 2005ip and $s=1.4-1.6$ for SN 2006jd).

The late phase LC of SN 2006jd shows an increase which is not expected in our model so we do not use the late time LC in the fit. This luminosity increase may be due to, e.g., 

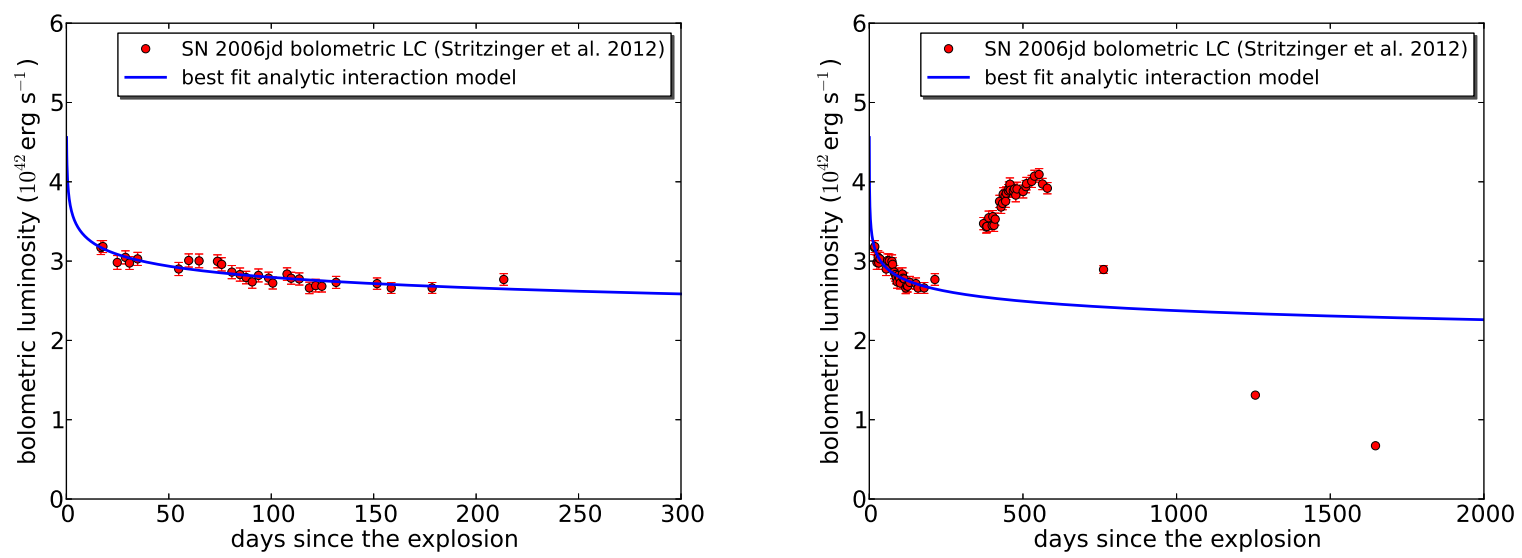

Figure 3. Bolometric LC of SN 2006jd (Stritzinger et al. 2012) and the best fit $L=L_{1} t^{\alpha}$ model up to 250 days. The second rise starting around 400 days cannot be explained by our model and may be due to, e.g., another CSM component.

another CSM component. Since we can only fit the early phases, we cannot constrain $M_{\mathrm{ej}}$ and $E_{\text {ej }}$ independently. Here, we assume $M_{\text {ej }}=10 M_{\odot}$ to estimate $E_{\text {ej }}$. The estimated $E_{\text {ej }}$ is $E_{\mathrm{ej}}= \begin{cases}1.2 \times 10^{52} \mathrm{erg} & (n=10), \\ 1.3 \times 10^{52} \mathrm{erg} & (n=12) .\end{cases}$

Note again that we assume $\epsilon=0.1$ (see Section 4.1). The time $t_{t}$ obtained by these values are

$t_{t}= \begin{cases}4.1 \times 10^{2} \text { days } & (n=10), \\ 1.8 \times 10^{2} \text { days } & (n=12) .\end{cases}$

The epochs we used to fit $L=L_{1} t^{\alpha}(t<250$ days $)$ are justified for the $n=10$ case. For the $n=12$ case, $t_{t}$ is smaller than 250 days. However, there are only two observational data points beyond 180 days and we find that the results of fitting by using $t<180$ days are almost the same as the results we obtained with $t<250$ days.

The CSM properties we derived are consistent with $s \simeq 1.5-1.6$ and the CSM density $\sim 10^{6} \mathrm{~cm}^{-3}$ at $\sim 2 \times 10^{16} \mathrm{~cm}$ as obtained by Chandra et al. (2012a) from X-ray and radio observations. However, the X-ray and radio observations were performed after the epochs when the bolometric LC starts to rise (after about 400 days since the explosion). Our model is not applicable at these epochs as is discussed above and this correspondence can be a coincidence.

\subsubsection{SN 2010jl}

SN 2010jl has been extensively observed in a wide range of wavelengths (Smith et al. 2011b, 2012; Stoll et al. 2011; Andrews et al. 2011; Chandra et al. 2012b; Fox et al. 2013; Maeda et al. 
2013). Zhang et al. (2012) obtained a bolometric LC of SN 2010jl based on their optical observations and we use it for our model comparison. Note that they do not have near-infrared observations and the bolometric LC is constructed without them. The date of the explosion is set to 12 days before the $V$-band LC peak reported by Stoll et al. (2011). We apply our spherically symmetric bolometric LC model but the observation of polarization indicates an asymmetric nature of the CSM around SN 2010jl (Patat et al. 2011).

At first, we use $L=L_{1} t^{\alpha}$ to fit the bolometric LC and get

$L=2.04 \times 10^{44}\left(\frac{t}{1 \text { day }}\right)^{-0.486} \operatorname{erg~s}^{-1}$

$\alpha=-0.486$ corresponds to $s=2.2$ and $s=2.3$ for $n=10$ and $n=12$, respectively. However, $t_{t}$ become

$t_{t}= \begin{cases}9.5 \text { days } & (n=10), \\ 3.8 \text { days } & (n=12),\end{cases}$

for $E_{\mathrm{ej}}=10^{52} \mathrm{erg}$ or

$t_{t}= \begin{cases}57 \text { days } & (n=10), \\ 23 \text { days } & (n=12),\end{cases}$

for $E_{\mathrm{ej}}=2.5 \times 10^{52} \mathrm{erg}$ with the obtained $L_{1}$. This means that the $L=L_{1} t^{\alpha}$ formula we used for the fitting is not applicable for most of the epochs we used for the fitting. Thus, we need to use the asymptotic formula (Equation 26) to fit the LC.

In Figure 4, we show some asymptotic LC models from Equation (26). We have searched for a good fit by changing $s, D$, and $E_{\mathrm{ej}}$. We assume $M_{\mathrm{ej}}=10 M_{\odot}$. The best model we found is shown in Figure 4 and it has

$\rho_{\mathrm{csm}}(r)=2.5 \times 10^{-14}\left(\frac{r}{10^{15} \mathrm{~cm}}\right)^{-2.2} \mathrm{~g} \mathrm{~cm}^{-3}$

and

$E_{\text {ej }}=2.3 \times 10^{52} \mathrm{erg}$.

The Thomson scattering optical depth of the solar-metallicity unshocked CSM when the shell is at $10^{15} \mathrm{~cm}$ is $\tau_{\mathrm{sh}}=7.1$. $\tau_{\mathrm{sh}}$ becomes $\sim 1$ at $\sim 5 \times 10^{15} \mathrm{~cm}$ and the shell is above $\sim 5 \times 10^{15} \mathrm{~cm}$ at the epochs we apply our model (after about 30 days since the explosion). The average mass-loss rate estimated by the CSM mass within $10^{16} \mathrm{~cm}$ for $v_{\mathrm{w}}=100 \mathrm{~km} \mathrm{~s}^{-1}$ is

$\langle\dot{M}\rangle=0.039 M_{\odot} \mathrm{yr}^{-1}$ 


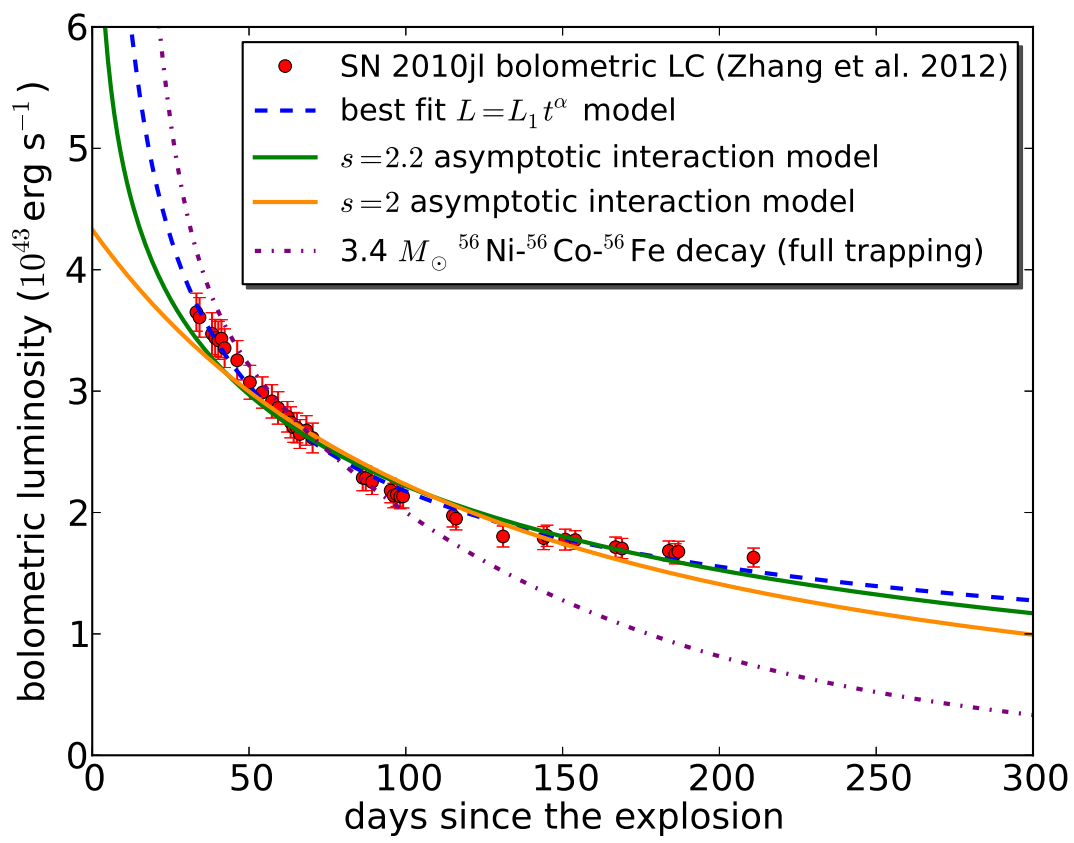

Figure 4. Bolometric LC of SN 2010jl (Zhang et al. 2012) and some model fits to it. The dashed line represents the best fit for $L=L_{1} t^{\alpha}$. However, $t_{t}$ expected from the result of the fit is too small to apply this model to the entire LC. Thus, we need to apply the asymptotic LC formula which is applicable after $t_{t}$. We get a good fit with $s=2.2$ (green line). The orange line is the best fit from the $s=2$ model. The dot-dashed line is the radioactive decay energy available from $3.4 M_{\odot}{ }^{56} \mathrm{Ni}$. The radioactive decay model is suggested by Zhang et al. (2012) to explain the early bolometric LC but our interaction model can explain the entire LC with a single component.

The estimated rate is consistent with those obtained based on the infrared emission (Maeda et al. 2013; Fox et al. 2013). $t_{t}=29$ days for $n=10$ and $\delta=1$ and $t_{t}=15$ days for $n=12$ and $\delta=1$. Thus the usage of the asymptotic formula is justified.

Since $s$ obtained above is close to the case of the steady mass loss $(s=2)$, we also try to fit the bolometric LC by the asymptotic formula $L=a(1+b t)^{-3 / 2}$ for $s=2$ (Equation 33). We obtain $a=4.38 \times 10^{43} \mathrm{erg} \mathrm{s}^{-1}$ and $b=6.44 \times 10^{-8} \mathrm{~s}^{-1}$ with $t_{t}=22$ days $(n=10$ and $\delta=1)$ or $t_{t}=13$ days $(n=12$ and $\delta=1)$. By using $a, b$, and $\epsilon=0.1$, we get

$E_{\mathrm{ej}}=1.4 \times 10^{52} \mathrm{erg}$,

from Equation (35). Assuming $M_{\mathrm{ej}}=10 M_{\odot}$ and $v_{w}=100 \mathrm{~km} \mathrm{~s}^{-1}$, we obtain

$\dot{M}=0.087 M_{\odot} \mathrm{yr}^{-1}$,

from Equation (36). The rate is similar to the average rate from the $s=2.2$ model derived above.

Comparing the $s=2.2$ and $s=2$ models, we find that the $s=2$ model has flatter LC than the $s=2.2$ model. As we make $s$ smaller, the model LC gets flatter and it gets harder to explain the bolometric LC of SN 2010jl. Thus, we presume that the CSM around 
the progenitor of SN 2010jl may be a bit steeper than the CSM expected from steady mass loss. This conclusion contradicts that obtained by Chandra et al. (2012b) from Xray observations. Chandra et al. (2012b) suggest $s=1.6$ for SN 2010jl based on X-ray observations. However, their estimate is obtained by assuming $r_{\mathrm{sh}} \propto t^{(n-3) /(n-s)}$ which is not likely applicable at the epochs when they obtained X-ray data $(\simeq 60$ days and $\simeq 360$ days since the explosion). This is because of the small $t_{t}$ mainly due to the high CSM density as is shown above.

So far, we fit the entire bolometric LC up to about 200 days by a single component. On the contrary, Zhang et al. (2012) suggested a two-componet model for the bolometric LC. They suggested that the LC before around 100 days is mainly powered by $3.4 M_{\odot}$ of ${ }^{56} \mathrm{Ni}$ whose available radioactive energy is shown in Figure 4. They suggested that the SN ejecta-CSM interaction started playing a role at later epochs by using a model LC of the interaction developed by Wood-Vasey, Wang, \& Aldering (2004). However, the required ${ }^{56} \mathrm{Ni}$ mass is very large $\left(3.4 M_{\odot}\right)$ and this amount of ${ }^{56} \mathrm{Ni}$ is rather difficult to produce in a core-collapse SN explosion (e.g., Umeda \& Nomoto 2008). In addition, no signatures of Fe elements are observed in the late phase spectra of SN 2010jl which are expected if there is large amount of ${ }^{56} \mathrm{Ni}$ production (e.g., Dessart et al. 2013). As noted in Section 3.2.1, the bolometric LC powered by the interaction resembles to the LC powered by the radioactive decay of ${ }^{56} \mathrm{Ni}$ at early epochs and we need to use additional late-phase LCs to distinguish between the two power sources. We have shown here that we need only one component from the interaction model to explain the whole LC of SN 2010jl.

\section{DISCUSSION}

\subsection{Sensitivity to the Assumed Parameters}

We have fixed $\epsilon=0.1$ and $M_{\mathrm{ej}}=10 M_{\odot}$ in deriving some SN ejecta and CSM properties in the previous section. Here, we discuss how sensitive the derived properties are to the assumed values of $\epsilon$ and $M_{\mathrm{ej}}$. In addition, it is practically difficult determine $v_{1}$ observationally from spectra of SNe IIn because the origins of the spectral features are not understood well. We also discuss the effect of the uncertainty in $v_{1}$.

At first, we assume that $v_{1}$ is well-determined and see the effect of $\epsilon$. The estimated CSM density structures or the estimated mass-loss rates depend on $\epsilon$ for a given $v_{1}$ (Equation 401). The average mass-loss rates have a relation $\langle\dot{M}\rangle \propto \epsilon^{-1}$. For example, we obtained 
$\langle\dot{M}\rangle=1.2 \times 10^{-3} M_{\odot} \mathrm{yr}^{-1}$ for SN 2005ip $(n=10)$ in the previous section by assuming $\epsilon=0.1$ but this mass-loss rate may be reduced to $\langle\dot{M}\rangle=2.4 \times 10^{-4} M_{\odot} \mathrm{yr}^{-1}$ if we assume $\epsilon=0.5$. This uncertainty makes it difficult to distinguish the RSG progenitor and the LBV progenitor. The assumption of $M_{\mathrm{ej}}=10 M_{\odot}$ is used to estimate $E_{\mathrm{ej}}$. $E_{\mathrm{ej}}$ depends on both $M_{\mathrm{ej}}$ and $\epsilon$ as $E_{\mathrm{ej}} \propto \epsilon^{-\frac{2}{n-3}} M_{\mathrm{ej}}^{\frac{n-5}{n-3}}$. For the typical values of $n$, we obtain $E_{\mathrm{ej}} \propto \epsilon^{-0.29} M_{\mathrm{ej}}^{0.71}$ $(n=10)$ or $E_{\text {ej }} \propto \epsilon^{-0.22} M_{\mathrm{ej}}^{0.78}(n=12)$. In either case, $E_{\mathrm{ej}}$ is mostly determined by the assumed $M_{\mathrm{ej}}$. For the SN 2005ip model $(n=10)$ in the previous section $\left(E_{\mathrm{ej}}=1.3 \times 10^{52}\right.$ erg for $\epsilon=0.1$ and $\left.M_{\mathrm{ej}}=10 M_{\odot}\right), E_{\mathrm{ej}}$ can be changed to, e.g., $7.9 \times 10^{51} \mathrm{erg}(\epsilon=0.1$ and $\left.M_{\mathrm{ej}}=5 M_{\odot}\right), 8.2 \times 10^{51} \mathrm{erg}\left(\epsilon=0.5\right.$ and $\left.M_{\mathrm{ej}}=10 M_{\odot}\right)$, or $5.0 \times 10^{51} \mathrm{erg}(\epsilon=0.5$ and $\left.M_{\mathrm{ej}}=5 M_{\odot}\right)$. Thus, the estimated $E_{\mathrm{ej}}$ are not much affected by the assumed parameters.

We have assumed that $v_{1}$ can be determined by spectral observations. However, spectra of SNe IIn have complicated features with several components and it is not obvious which spectral component originates from the dense shell and can be used to estimate $v_{1}$. Thanks to the formation of the dense shell due to the radiative cooling, the shell velocity is one of the fastest components in the system (see also Section 4.4). Thus, we have used the fastest velocity component in the spectra to estimate $v_{1}$ in the previous section $\left(17,500 \mathrm{~km} \mathrm{~s}^{-1}\right.$ at 100 days for SN 2005ip). Indeed, $\langle\dot{M}\rangle \propto v_{1}^{s-5}\left(\langle\dot{M}\rangle \propto v_{1}^{-3}\right.$ for $\left.s \simeq 2\right)$ and the estimated mass-loss rates are rather sensitive to the assumed $v_{1}$. Keeping $\epsilon=0.1$ and $M_{\mathrm{ej}}=10 M_{\odot}$, we obtain $\langle\dot{M}\rangle=1.8 \times 10^{-3} M_{\odot} \mathrm{yr}^{-1}$ and $E_{\text {ej }}=1.0 \times 10^{52} \mathrm{erg}$ for $v_{\mathrm{sh}}(100$ days $)=15,000 \mathrm{~km} \mathrm{~s}^{-1}$, $\langle\dot{M}\rangle=5.5 \times 10^{-3} M_{\odot} \mathrm{yr}^{-1}$ and $E_{\text {ej }}=5.8 \times 10^{51}$ erg for $v_{\mathrm{sh}}(100$ days $)=10,000 \mathrm{~km} \mathrm{~s}^{-1}$, and $\langle\dot{M}\rangle=3.6 \times 10^{-2} M_{\odot} \mathrm{yr}^{-1}$ and $E_{\mathrm{ej}}=2.2 \times 10^{51} \mathrm{erg}$ for $v_{\mathrm{sh}}(100$ days $)=5,000 \mathrm{~km} \mathrm{~s}^{-1}$. As we use the highest velocity component to estimate $v_{1}$ in the previous section, the estimated mass-loss rates were rather conservative. Note again that the dense shell velocity should be one of the fastest components in the system we model (see also Section 4.4) and thus adopting the higher observed velocity is preferred.

Finally, $v_{\mathrm{w}}$ has been assumed to be $100 \mathrm{~km} \mathrm{~s}^{-1}$, which is a typical LBV wind velocity (e.g., Leitherer 1997). SN IIn spectra often show a $100 \mathrm{~km} \mathrm{~s}^{-1}$ P-Cygni profile. The massloss rates estimated are proportional to $v_{\mathrm{w}}$. If SNe IIn are from RSGs, the wind velocity can be lower (e.g., Mauron \& Josselin 2011) and the mass-loss rates estimated will be decreased because of the lower wind velocities. 


\subsection{Applicability}

In deriving the evolution of the shocked-shell radius $r_{\mathrm{sh}}(t)$, we have assumed that $s$ is smaller than 3. This condition is also required to derive a physical self-similar solution (e.g., Nadyozhin 1985). The allowed range of $\alpha$ for $s<3$ is $\alpha>-1$ because $\alpha \rightarrow-1(s \rightarrow 3)$ and $\alpha$ is a monotonically-decreasing function at $n>5$. Thus, if we obtain $\alpha<-1$ by fitting $L=L_{1} t^{\alpha}$, this is beyond the applicability of our model and we need to consider other ways to explain the LC.

First, we need to check $t_{t}$. If $t_{t}$ is smaller than the time used for the fitting, we need to use the asymptotic formula for the fitting. The asymptotic formula can have a rapid decline in the bolometric LC depending on parameters.

Another possibility is a CSM with $s>3$. Most of the mass in CSM with $s>3$ exists near the inner edge of the CSM. In other words, for the case of $s>3$,

$$
\begin{aligned}
M_{\mathrm{csm}} & \equiv \int_{R_{p}}^{r_{\mathrm{sh}}} 4 \pi r^{2} \rho_{\mathrm{csm}} d r \\
& =\frac{4 \pi D}{s-3}\left(R_{p}^{3-s}-r_{\mathrm{sh}}^{3-s}\right) \\
& \simeq \frac{4 \pi D}{s-3} R_{p}^{3-s}=\text { constant }\left(r_{\mathrm{sh}} \gg R_{p}\right)
\end{aligned}
$$

Thus, most of the CSM is shocked soon after the explosion. If the CSM density is relatively low, the LCs will decline quickly soon after the explosion when most of the CSM component is swept up. If the shocked shell becomes optically thick, LCs may resemble the 'shell-shocked diffusion' model LC suggested by Smith \& McCray (2007) as a model for superluminous SNe based on the formalisms by Arnett (1980) (but see also Moriva et al. 2013a). This is a LC model for the declining part of the bolometric LC after the shock wave passes through a dense CSM. According to this model, the declining part of the bolometric LCs follows

$L=L_{0} \exp \left[-\frac{t}{\tau_{\text {diff }}}\left(1+\frac{t}{2 \tau_{\exp }}\right)\right]$,

where $t$ is the time since the maximum luminosity, $\tau_{\text {diff }}$ is the characteristic diffusion timescale in the shocked shell and $\tau_{\exp }$ is the expansion timescale of the shocked shell.

Bolometric LCs can also follow Equation (65) even if $s<3$. This is the case when the high-density CSM is small in radius and the entire high-density CSM is shocked soon after the beginning of the interaction. Then, there is no continuous interaction and the bolometric LC should decline quickly, possibly following the shell-shocked diffusion model. However, in 
this case, there may be little remaining CSM to emit narrow emission lines in spectra and the SN may not continue to be of Type IIn.

So far, we have only considered possible ways to understand rapidly declining LCs in the context of the SN ejecta-dense CSM interaction. For the case of SNe IIn, it is natural to consider in the context of the interaction model. However, it is possible that CSM around some SNe IIn are dense enough only to affect their spectra while their LCs are not much affected by the dense CSM. Then, rapidly declining LCs may be powered by other mechanisms like ${ }^{56} \mathrm{Ni}$, magnetars (e.g., Maeda et al. 2007; Kasen \& Bildsten 2010; Wooslev 2010), or fallback (Dexter \& Kasen 2012).

\subsection{Initial Luminosity Increase}

The bolometric LC model presented in this paper does not have a rising part at the beginning. There are several mechanisms to make the initial luminosity increase in LCs which are not taken into account in our model.

We have assumed that the radiation emitted from the dense shell is not affected by the unshocked CSM. However, especially at the early phases just after the explosion, the CSM surrounding the dense shell can be optically thick and the radiation from the shell can be scattered within the CSM. In this case, the diffusion timescale in the optically thick region determines the evolution of the initial luminosity increase and subsequent decline. Our model should only be applied to the epochs when the CSM surrounding the dense shell becomes optically thin and should not be applied at the epochs when the luminosity increases or just after the luminosity peaks. When the CSM is optically thick, some signatures can be seen in spectra as well (e.g., Chugai 2001).

If the CSM is optically thin, the timescale of the initial luminosity increase is expected to be very small. Two mechanisms can affect the initial luminosity increase. One is the shock breakout at the surface of the progenitor and the other is the on-set of the SN ejecta-CSM interaction. Both are presumed to have a short timescale. If the dense part of the CSM and the progenitor are detached, we may see two luminosity peaks in the early phases: one from the shock breakout and the other from the on-set of the interaction. 

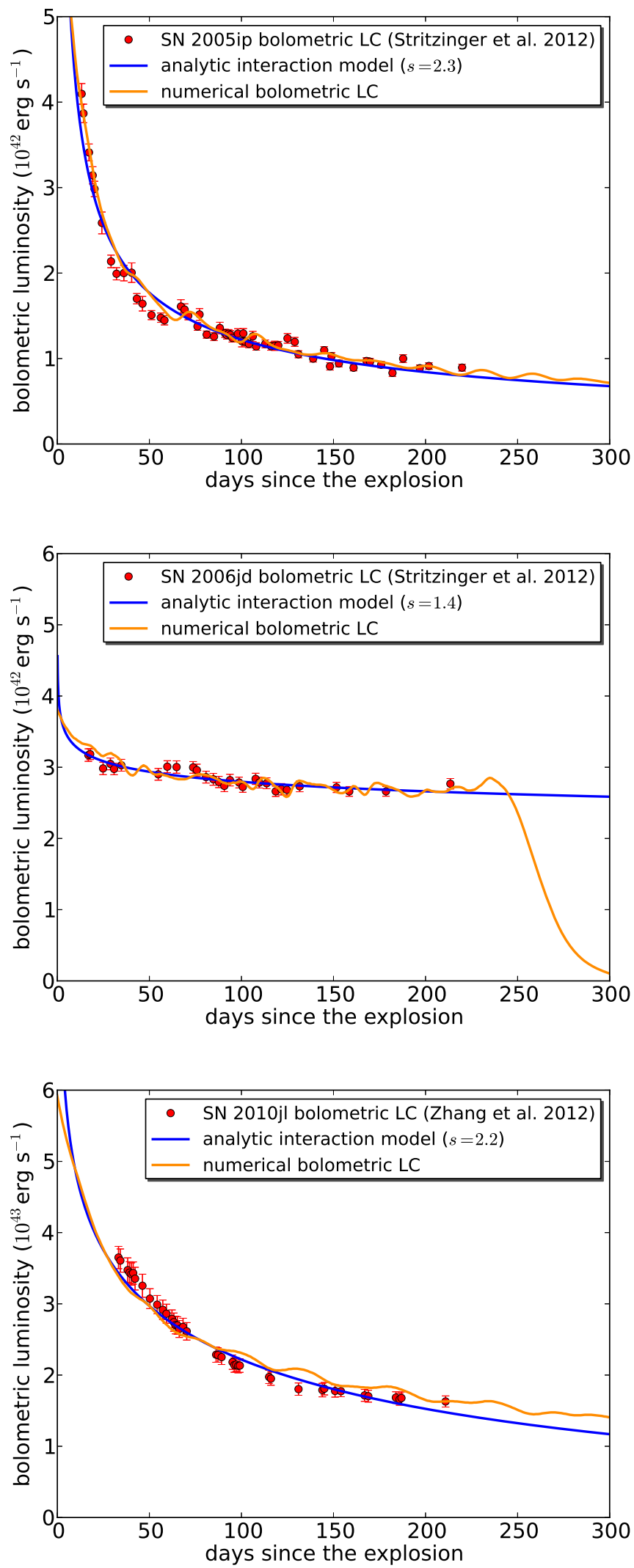

Figure 5. Comparisons between the numerical bolometric LCs and the analytic bolometric LCs presented in Section 3.2 based on which the initial conditions for the numerical bolometric LC computations are constructed. 

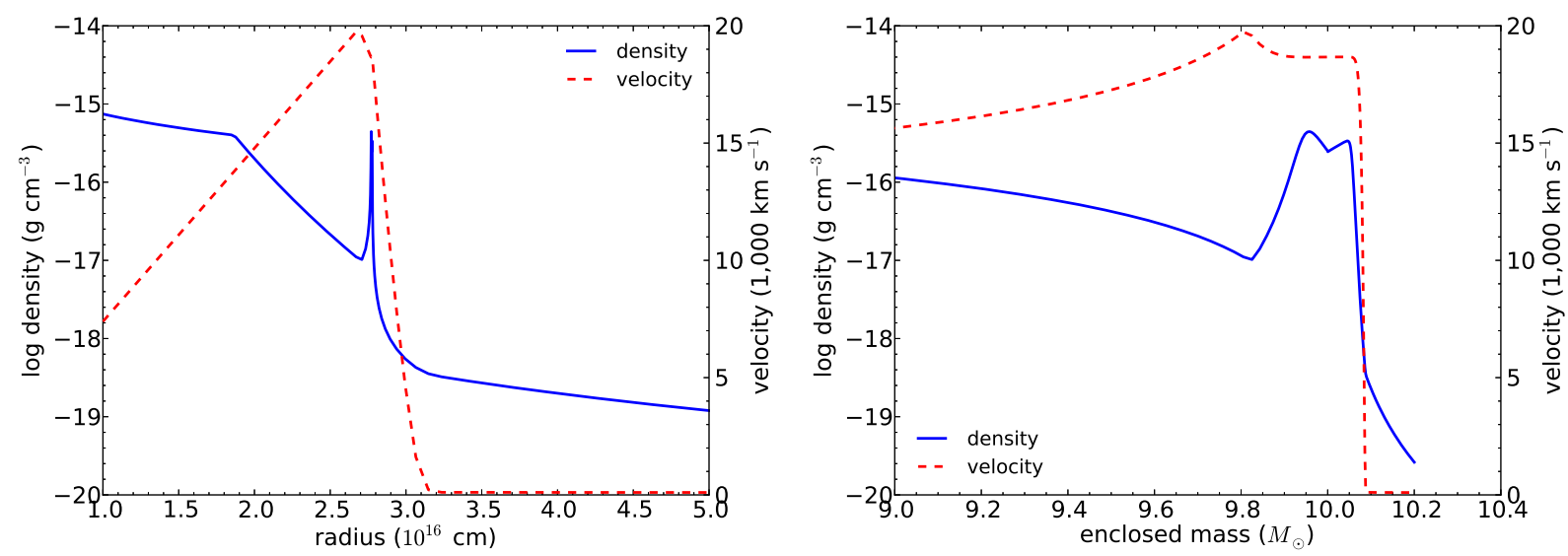

Figure 6. Density and velocity structures of the numerical model for SN 2005ip at 100 days.

\subsection{Comparison with Numerical Bolometric Light Curves}

To show the reliability of our analytic LC model, we also performed numerical LC calculations using a one-dimensional radiation hydrodynamics code STELLA (e.g., Blinnikov \& Bartunov 1993; Blinnikov et al. 2000, 2006). We show some comparisons in this paper and more detailed comparisons will be presented elsewhere. We set the initial conditions following the physical parameters obtained in Section 3.2. The density structure of the homologously expanding SN ejecta has two power-law components as is assumed in the analytic model. The SN ejecta and CSM are initially connected at $10^{14} \mathrm{~cm}$. The CSM outer radius of all the models is set to $10^{17} \mathrm{~cm}$. The parameter $B_{q}$ which controls the conversion efficiency from the kinetic energy to radiation in the code (see Moriva et al. 2013b for the details) is adjusted to make $\epsilon \simeq 0.1$. Both SN ejecta and CSM in the calculations have solar composition.

Figure 5 presents the results of our LC calculations. We performed the LC calculations of three models in Section [3.2, namely, SN 2005ip $\left(s=2.3, n=10, \delta=1, E_{\text {ej }}=1.2 \times 10^{52}\right.$ $\mathrm{erg}$, and $\left.M_{\mathrm{ej}}=10 M_{\odot}\right)$, SN 2006jd $\left(s=1.4, n=10, \delta=1, E_{\mathrm{ej}}=1.3 \times 10^{52} \mathrm{erg}\right.$, and $\left.M_{\mathrm{ej}}=10 M_{\odot}\right)$, and SN 2010jl $\left(s=2.2, n=10, \delta=1, E_{\mathrm{ej}}=2.3 \times 10^{52} \mathrm{erg}\right.$, and $\left.M_{\mathrm{ej}}=10 M_{\odot}\right)$.

The overall features of the analytic LCs are well reproduced by the numerical LCs and the analytic model presented in this paper is shown to provide a good prediction to the numerical results.

In Figure 6, we show the density and velocity structures of the numerical SN 2005ip model at 100 days in radius and mass coordinates. We can see that the dense shell is formed between the SN ejecta and the dense CSM and the shell width is much smaller than the shell radius because of the radiative cooling. The plot in the mass coordinate indicates that (C) 2013 RAS, MNRAS 000, 1,29 
most of the shocked SN ejecta and CSM is in this thin shell. Thus the assumption in our analytic model that the shocked region can be expressed by using a single $r_{\text {sh }}$ is verified. This means that the forward and reverse shocks are glued to the cool dense shell and the velocities of them are not different from each other so much at these early epochs because of the radiative cooling. Note that the shell is one of the fastest velocity components at this epoch and the shell velocity is consistent with $v_{1}$ adopted $\left(v_{\mathrm{sh}} \simeq 17,500 \mathrm{~km} \mathrm{~s}^{-1}\right.$ at 100 days). We can also see that the density structure ahead of the shell is modified slightly because of the precursor.

\subsection{Non-Bolometric Light Curves}

As our LC model takes only the sum of the available energy into account, the LC we obtain from the model is bolometric and we have applied our analytic bolometric LC model to bolometric LCs constructed from observations. Here we try to fit the $L=L_{1} t^{\alpha}$ formula to optical and near-infrared LCs of SN 2005ip and SN 2006jd obtained by Stritzinger et al. (2012). We focus on the parameter $\alpha$ which is directly affected by the CSM density slope $s$ for a given $n$.

Figure 7 and Table 2 show the results of the LC fits. As we can see, $\alpha$ obtained with different photometric bands have different values. This means that we need to construct a bolometric LC from observations to obtain accurate information. This can be clearly seen in Figure 7 of Stritzinger et al. (2012). The spectra evolve significantly with time and no single band can represent the entire evolution of the bolometric LC. We thus clearly need to construct a bolometric LC to apply our model to obtain CSM and SN properties of SNe IIn.

\section{CONCLUSIONS}

We have developed an analytic bolometric LC model for SNe powered by the interaction between SN ejecta and dense CSM. This model is suitable for modeling SNe IIn. We have analytically derived the evolution of the shocked dense shell created by the interaction. We obtain the bolometric LC evolution from the derived dense shell evolution. Our model is not restricted to the CSM from steady mass loss.

We have applied our bolometric LC model to three SNe IIn whose bolometric LCs have been constructed from observations, i.e., SN 2005ip, SN 2006jd, and SN 2010jl. The results 
Table 2. List of $\alpha$ from optical and near-infrared LCs.

\begin{tabular}{ccc}
\hline Band & \multicolumn{2}{c}{$\alpha$} \\
& SN 2005ip & SN 2006jd \\
\hline bolometric & -0.536 & -0.0708 \\
$u$ & -1.01 & -0.300 \\
$B$ & -0.923 & -0.374 \\
$g$ & -0.934 & -0.387 \\
$V$ & -0.995 & -0.451 \\
$r$ & -0.854 & -0.557 \\
$i$ & -1.00 & -0.592 \\
$Y$ & -0.706 & -0.414 \\
$J$ & -0.630 & -0.137 \\
$H$ & -0.171 & 0.0950 \\
\hline
\end{tabular}

show that their CSM density slopes are close to what is expected from the steady mass loss $\left(s=2\right.$ where $\left.\rho_{\text {csm }} \propto r^{-s}\right)$ but slightly deviate from it $(s \simeq 2.3-2.4$ for SN 2005ip, $s \simeq 1.4-1.6$ for SN 2006jd, and $s \simeq 2.2$ for SN 2010jl). The derived mass-loss rates are consistent with LBVs (SN 2005ip: $\langle\dot{M}\rangle=0.0012-0.0014 M_{\odot} \mathrm{yr}^{-1}$, SN 2006jd: $\langle\dot{M}\rangle=$ $0.0013-0.0017 M_{\odot} \mathrm{yr}^{-1}$ and SN 2010jl: $\langle\dot{M}\rangle=0.039 M_{\odot} \mathrm{yr}^{-1}$ ). We could not constrain SN ejecta properties strongly but $E_{\text {ej }}$ of all three SNe likely exceeded $10^{52}$ erg if we assume that $M_{\mathrm{ej}}=10 M_{\odot}$ and that the conversion efficiency from kinetic energy to radiation is $10 \%$ $(\epsilon=0.1)$.

We have also found that the energy inputs from the interaction and the radioactive decay of ${ }^{56} \mathrm{Ni}$ can be similar to each other up to about 100 days since the explosion. We need to have LCs also at later phases to distinguish between the two luminosity sources from LCs alone.

Our bolometric LC model can only be applied for $s<3$. For $s>3$, we suggest that the shell-shocked diffusion model proposed by Smith \& McCray (2007) (see also Moriya et al. 2013a) may be applied for some cases.

We have also compared our analytic LCs to synthetic ones calculated with a onedimensional radiation hydrodynamics code STELLA. Our analytic LCs are well-reproduced by the numerical modeling.

We have applied our model to only three observed SNe IIn. We suggest to systematically study SN ejecta and CSM properties of SNe IIn by applying our LC model to many other SNe IIn. Such a systematic study will lead to a comprehensive understanding of SNe IIn, i.e., their progenitors and the mass-loss mechanisms related to them. 

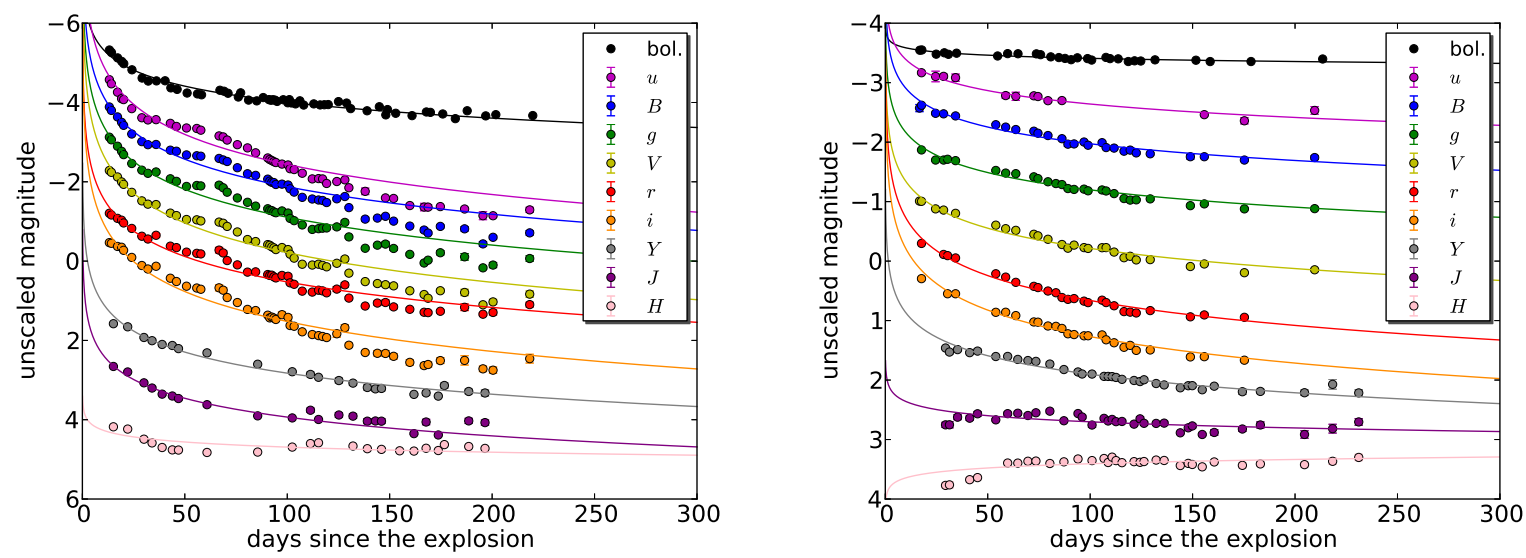

Figure 7. Multi-color LCs of SN 2005ip and SN 2006jd and the results of the fit to $L \propto t^{\alpha}$. $\alpha$ obtained by multi-color LCs are not consistent with $\alpha$ obtained from the bolometric LC. We need a bolometric LC to infer CSM and SN ejecta properties from LCs properly.

\section{ACKNOWLEDGMENTS}

We thank the anonymous referee for the comments which improved this paper. T.J.M. and K.M. thank the staff at Stockholm University for their hospitality during their stay as shortterm visitors when this project was initiated. T.J.M. is supported by the Japan Society for the Promotion of Science Research Fellowship for Young Scientists (23.5929). K.M. acknowledges the financial support by a Grant-in-Aid for Scientific Research for Young Scientists (23740141). This research is also supported by World Premier International Research Center Initiative, MEXT, Japan. The Oskar Klein Centre is funded by the Swedish Research Council. The work in Russia was supported by RF Government grant 11.G34.31.0047, grants for supporting Scientific Schools 5440.2012.2 and 3205.2012.2, and joint RFBR-JSPS grant 13-02-92119.

\section{REFERENCES}

Anderson J. P., Habergham S. M., James P. A., Hamuy M., 2012, MNRAS, 424, 1372

Andrews J. E., et al., 2011, AJ, 142, 45

Arnett W. D., 1980, ApJ, 237, 541

Blinnikov S. I., Bartunov O. S., 1993, A\&A, 273, 106

Blinnikov S., Lundqvist P., Bartunov O., Nomoto K., Iwamoto K., 2000, ApJ, 532, 1132

Blinnikov S., Potashov M., Baklanov P., Dolgov A., 2012, JETPL, 96, 153

Blinnikov S. I., Röpke F. K., Sorokina E. I., Gieseler M., Reinecke M., Travaglio C., Hillebrandt W., Stritzinger M., 2006, A\&A, 453, 229 
Botticella M. T., et al., 2009, MNRAS, 398, 1041

Chandra P., Chevalier R. A., Chugai N., Fransson C., Irwin C. M., Soderberg A. M., Chakraborti S., Immler S., 2012a, ApJ, 755, 110

Chandra P., Chevalier R. A., Irwin C. M., Chugai N., Fransson C., Soderberg A. M., 2012b, ApJ, 750, L2

Chatzopoulos E., Wheeler J. C., Vinko J., 2012, ApJ, 746, 121

Chatzopoulos E., Wheeler J. C., Vinko J., Horvath Z. L., Nagy A., 2013, arXiv, arXiv:1306.3447

Chevalier R. A., 2012, ApJ, 752, L2

Chevalier R. A., 1990, supe.conf, 91

Chevalier R. A., 1982a, ApJ, 259, 302

Chevalier R. A., 1982b, ApJ, 258, 790

Chevalier R. A., Fransson C., 2006, ApJ, 651, 381

Chevalier R. A., Fransson C., 2003, LNP, 598, 171

Chevalier R. A., Fransson C., 1994, ApJ, 420, 268

Chevalier R. A., Fransson C., Nymark T. K., 2006, ApJ, 641, 1029

Chevalier R. A., Irwin C. M., 2012, ApJ, 747, L17

Chevalier R. A., Irwin C. M., 2011, ApJ, 729, L6

Chugai N. N., 2001, MNRAS, 326, 1448

Chugai N. N., Danziger I. J., 1994, MNRAS, 268, 173

Chugai N. N., et al., 2004, MNRAS, 352, 1213

Cooke J., 2008, ApJ, 677, 137

Cooke J., Sullivan M., Barton E. J., Bullock J. S., Carlberg R. G., Gal-Yam A., Tollerud E., 2009, Natur, 460, 237

Cooke J., et al., 2012, Natur, 491, 228

Crowther P. A., 2013, MNRAS, 428, 1927

Crowther P. A., 2007, ARA\&A, 45, 177

Dessart L., Waldman R., Livne E., Hillier D. J., Blondin S., 2013, MNRAS, 428, 3227

Dexter J., Kasen D., 2012, arXiv, arXiv:1210.7240

Dwarkadas V. V., 2011, MNRAS, 412, 1639

Dwarkadas V. V., Gruszko J., 2012, MNRAS, 419, 1515

Filippenko A. V., 1997, ARA\&A, 35, 309

Fox O. D., Filippenko A. V., Skrutskie M. F., Silverman J. M., Ganeshalingam M., Cenko (C) 2013 RAS, MNRAS 000, 1,29 
S. B., Clubb K. I., 2013, AJ, 146, 2

Fox O. D., et al., 2011, ApJ, 741, 7

Fox O. D., Chevalier R. A., Dwek E., Skrutskie M. F., Sugerman B. E. K., Leisenring J. M., 2010, ApJ, 725, 1768

Fox O., et al., 2009, ApJ, 691, 650

Fransson C., Lundqvist P., Chevalier R. A., 1996, ApJ, 461, 993

Gal-Yam A., Leonard D. C., 2009, Natur, 458, 865

Ginzburg S., Balberg S., 2012, ApJ, 757, 178

Groh J. H., Meynet G., Ekström S., 2013, A\&A, 550, L7

Heger A., Fryer C. L., Woosley S. E., Langer N., Hartmann D. H., 2003, ApJ, 591, 288

Kasen D., 2010, ApJ, 708, 1025

Kasen D., Bildsten L., 2010, ApJ, 717, 245

Kiewe M., et al., 2012, ApJ, 744, 10

Kozasa T., Nozawa T., Tominaga N., Umeda H., Maeda K., Nomoto K., 2009, ASPC, 414, 43

Langer N., 2012, ARA\&A, 50, 107

Maeda K., 2013, ApJ, 762, 14

Maeda K., et al., 2013, ApJ, submitted

Maeda K., et al., 2007, ApJ, 666, 1069

Maeder A., Desjacques V., 2001, A\&A, 372, L9

Maeder A., Meynet G., 2000, A\&A, 361, 159

Matzner C. D., McKee C. F., 1999, ApJ, 510, 379

Mauerhan J. C., et al., 2013, MNRAS, 749

Mauron N., Josselin E., 2011, A\&A, 526, A156

Moriya T. J., Blinnikov S. I., Baklanov P. V., Sorokina E. I., Dolgov A. D., 2013a, MNRAS, 430, 1402

Moriya T. J., Blinnikov S. I., Tominaga N., Yoshida N., Tanaka M., Maeda K., Nomoto K., 2013b, MNRAS, 428, 1020

Moriya T. J., Groh J. H., Meynet G., 2013, arXiv, arXiv:1306.0605

Moriya T. J., Tominaga N., 2012, ApJ, 747, 118

Nadyozhin D. K., 1994, ApJS, 92, 527

Nadyozhin D. K., 1985, Ap\&SS, 112, 225

Owocki S. P., Gayley K. G., Shaviv N. J., 2004, ApJ, 616, 525 
Pastorello A., et al., 2013, ApJ, 767, 1

Patat F., Taubenberger S., Benetti S., Pastorello A., Harutyunyan A., 2011, A\&A, 527, L6

Poelarends A. J. T., Herwig F., Langer N., Heger A., 2008, ApJ, 675, 614

Potashov M., Blinnikov S., Baklanov P., Dolgov A., 2013, MNRAS, L70

Prieto J. L., et al., 2008, ApJ, 681, L9

Quataert E., Shiode J., 2012, MNRAS, 423, L92

Leitherer C., 1997, ASPC, 120, 58

Schlegel E. M., 1990, MNRAS, 244, 269

Sakurai A., 1960, Commun. Pure Appl. Math., 13, 353

Smith N., Hinkle K. H., Ryde N., 2009, AJ, 137, 3558

Smith N., Silverman J. M., Filippenko A. V., Cooper M. C., Matheson T., Bian F., Weiner B. J., Comerford J. M., 2012, AJ, 143, 17

Smith N., Li W., Filippenko A. V., Chornock R., 2011a, MNRAS, 412, 1522

Smith N., et al., 2011b, ApJ, 732, 63

Smith N., et al., 2009, ApJ, 695, 1334

Smith N., McCray R., 2007, ApJ, 671, L17

Stoll R., Prieto J. L., Stanek K. Z., Pogge R. W., Szczygieł D. M., Pojmański G., Antognini J., Yan H., 2011, ApJ, 730, 34

Stritzinger M., et al., 2012, ApJ, 756, 173

Svirski G., Nakar E., Sari R., 2012, ApJ, 759, 108

Taddia F., et al., 2013, A\&A, 555, A10

Tanaka M., Moriya T. J., Yoshida N., 2013, arXiv, arXiv:1306.3743

Tanaka M., Moriya T. J., Yoshida N., Nomoto K., 2012, MNRAS, 422, 2675

Umeda H., Nomoto K., 2008, ApJ, 673, 1014

Whalen D. J., et al., 2013, ApJ, 768, 195

Wilms J., Allen A., McCray R., 2000, ApJ, 542, 914

Wood-Vasey W. M., Wang L., Aldering G., 2004, ApJ, 616, 339

Woosley S. E., 2010, ApJ, 719, L204

Woosley S. E., Blinnikov S., Heger A., 2007, Natur, 450, 390

Yoon S.-C., Cantiello M., 2010, ApJ, 717, L62

Zhang T., et al., 2012, AJ, 144, 131 